\title{
Efficient $\beta$-Carboline Alkaloid-Based Probe for Highly Sensitive Imaging of Endogenous Glutathione in Wheat Germ Tissues
}

\author{
Xiaohui Ji, ${ }^{1}$ Dan Zhang $\mathbb{D}^{1},{ }^{1}$ Le $\mathrm{Li}^{2}{ }^{2}$ Lingxia Jin, ${ }^{1}$ and Rui $\mathrm{Wu}{ }^{1}$ \\ ${ }^{1}$ Shaanxi Province Key Laboratory of Catalytic Foundation and Application, School of Chemistry and Environment Science, \\ Shaanxi University of Technology, Hanzhong 723001, China \\ ${ }^{2}$ Shaanxi Key Laboratory of Industrial Automation, School of Mechanical Engineering, Shaanxi University of Technology, \\ Hanzhong 723001, China \\ Correspondence should be addressed to Dan Zhang; zhangdan@snut.edu.cn
}

Received 28 November 2019; Revised 3 July 2020; Accepted 6 September 2020; Published 15 September 2020

Academic Editor: Neil D. Danielson

Copyright (c) 2020 Xiaohui Ji et al. This is an open access article distributed under the Creative Commons Attribution License, which permits unrestricted use, distribution, and reproduction in any medium, provided the original work is properly cited.

Discriminative detection of GSH is achieved by employing a highly sensitive and selective fluorescent probe (KL-DN) that bears $\beta$-carboline alkaloid as a potential fluorophore and an azide group as the recognition unit. A rapid fluorescence off-on change is caused by special redox reaction; KL-DN has the capability of monitoring endogenous GSH in wheat germ tissues, indicating that this probe holds great potential for biological applications in plant tissues.

\section{Introduction}

Wheat germ containing abundant GSH, a nutrition with high protein, high vitamin E, and low cholesterol, is beneficial to human health [1]. Interestingly, research indicates that GSH directly or indirectly involves in many functional activities in wheat seedling leaves under osmotic stress, including the metabolism of drugs and hormone in the plant cells and the biosynthesis of DNA, RNA, and protein [2-4]. As an essential endogenic antioxidant, GSH can protect plant cells from oxidative damage [5]. Accordingly, the development of economical and effective methods for detection of GSH in wheat germ system is of considerable importance for better understanding of its physiological functions and has become the significant subject of current chemical research.

Among the multiple analytical methods including electrochemical voltammetry, optical sensing, high-performance liquid chromatography, and mass spectrometry, fluorescence analysis is more popular because of its high safety, low cost, simplicity of operation, and noninvasiveness [6-8]. In consideration of the sensitivity and great bioimaging potential of fluorescence detection [9], considerable effort had been paid in the development of fluorescent probes for GSH detection based on different reactive mechanisms (Figure 1). For instance, Xie et al. introduced that the $\mathrm{SH}$ residue of GSH, and the aldehyde group of the fluorescent probe underwent the addition reaction resulting in the open-ring form in acid conditions [10]. Disulfide bonds have been abundantly exploited to measure GSH for mediating the extracellular release of drugs [11-14]. The disadvantages of long reaction time and high limit of detection were the ubiquitous challenge. The fluorescent probes with better SNAr leaving groups were susceptible to SNAr substitution by sulfhydryl thiols with poor selectivity [15-20]. Qu and coworkers reported a fluorescent turn-on method of Ag-S-GF taking advantage of specific interactions between sulfur and silver cation [21]. The mechanisms of recognizing GSH also included the selective cleavage of the selenium-nitrogen bond, Michael's addition, the reduction of azido, and others [22-30]. Although these researches greatly accelerated the development of fluorescent detection, the design of a highly sensitive chemosensor on the basis of natural product structure for recognizing GSH in situ in plant organisms is still a significant challenge.

As an active natural product, $\beta$-carboline alkaloids with good biocompatibility are composed of indole ring and 


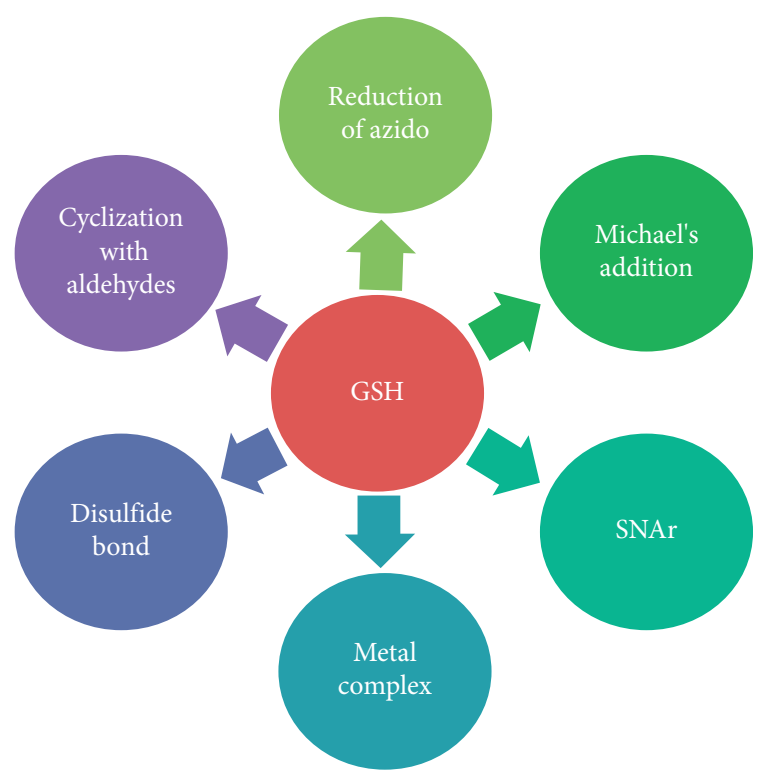

Figure 1: The reaction types of GSH.

pyridine ring, showing broad and significant biological activities [31-33]. In addition to these advantages, $\beta$-carboline alkaloids are capable of emitting strong fluorescence [34]. Unfortunately, very little research on using $\beta$-carboline alkaloids as fluorescent probes had been reported [35]. Selecting $\beta$-carboline alkaloid as a potential fluorophore and an azide group as the GSH recognition unit, we reported herein the design, synthesis, and evaluation of the turn-on probe KL-DN for rapidly discriminating GSH (Scheme 1). This novel probe offered exceedingly rapid ( $\sim 2 \mathrm{~min})$ and low concentration ( $~ 92 \mathrm{nM})$ (3s/slope) detection of GSH under physiological conditions without the need for excessively high probe/analyte ratios to achieve rapid probe activation. KL-DN exhibited remarkable selectivity toward GSH and Cys in comparison to other amino acids and the gasotransmitter $\mathrm{H}_{2} \mathrm{~S}$. Importantly, due to its design, KL-DN is taken up and activated in a very rapid fashion, so as to offer high-speed imaging of endogenous GSH in plant tissues and human cells with a high signal-to-background ratio.

\section{Results and Discussion}

2.1. Design and Synthesis of $K \mathbf{K}-\mathbf{D N}$. The KL-DN was prepared through the synthetic route outlined in Figure S1. We selected the inexpensive raw material (tryptophan) to synthesize compound $\mathbf{1}$ by Pictet-Spengler reaction. By means of oxidation reaction and nitrification, the formed compound 3 was changed into compound $\mathbf{4}$ in the presence of $\mathrm{Pd} / \mathrm{C}$ and hydrazine hydrate. Then the amine group of compound 4 was further converted to azide group of KL-DN with $53 \%$ yield. The final product was characterized by ${ }^{1} \mathrm{H}$ $\mathrm{NMR},{ }^{13} \mathrm{C}$ NMR, and MS spectrum (Figures S5-S7).

2.2. Evaluation of Optical Responses to GSH. The ability of KL-DN for sensing GSH was investigated in $10 \mathrm{mM}$ PBS buffer $(\mathrm{pH}=7.4)$ with $50 \%$ DMSO $(\mathrm{v} / \mathrm{v})$ at $25^{\circ} \mathrm{C}$. Under this condition, KL-DN $(10 \mu \mathrm{M})$ showed maximum absorption at $275 \mathrm{~nm}$ and displayed weak fluorescence. Upon addition of 2 eq GSH, the absorption intensity at $310 \mathrm{~nm}$ increased, which indicated that KL-DN was capable of reacting with GSH. According to the absorption spectrum, we choose $310 \mathrm{~nm}$ as the excitation wavelength. The fluorescence intensity at a new red-shifted emission peak $(487 \mathrm{~nm})$ evidently enhanced as high as 20-fold upon successive addition of GSH with good linear relationship and large stokes shifts (Figure 2(b)) (Figure S8A). The fluorescence quantum yield of KL-DN with the addition of GSH increased from 0.0013 to 0.038 with quinine sulfate as reference. The solvent system for measuring the fluorescence quantum yield of the probe KLDN was in DMSO/PBS buffer (1:1). Meanwhile, the solution started to show a bright blue fluorescence, which is highly visible to the naked eye (observed under a portable $365 \mathrm{~nm}$ UV light) (Figure S8B). The Job's plot through fluorescence intensity changes indicated a 1:1 reactive ratio of probe with GSH (Figure S9). To investigate the effects of $\mathrm{pH}$ on the fluorescent response of KL-DN toward GSH, fluorescence intensity changes were measured from $\mathrm{pH} 2-12$. In the presence of GSH, the probe KL-DN showed strong fluorescence in the range of 6-8 (Figure S10), which demonstrated that KL-DN could be used to detect GSH in real samples.

2.3. Selectivity of $\mathbf{K L}-\mathbf{D N}$ to GSH. Besides sensitivity, selectivity is another very important parameter to evaluate the performance of a new fluorescent probe in the presence of amino acids. Under the same conditions, the fluorescence changes of KL-DN upon addition of various amino acids and sulfide were almost negligible within $2 \mathrm{~min}$. Only GSH and Cys promoted fluorescent signal enhancement at $487 \mathrm{~nm}$ (Figure 3). Interestingly, KL-DN showed no fluorescence change when responding to the same amount of sulfide and Hcy. No absorption spectral change of KLDN added sulfide and Hcy was obtained (Figure S11). We tried to test fluorescence spectra of KL-DN $(10 \mu \mathrm{M})$ upon addition of excess $\mathrm{Na}_{2} \mathrm{~S}$ (50 eq) and $\mathrm{Hcy}$ (20 eq) for $45 \mathrm{~min}$. In fact, $\mathrm{Na}_{2} \mathrm{~S}$ and $\mathrm{Hcy}$ enabled reacting with azide to induce the generation of amine, but the reaction rate was too slow to interfere with GSH detection (Figures S12-S13). We also studied the NPA charges on $\mathrm{S}$ of $\bullet \mathrm{OH}$ for Path R4 in the gas (a) and aqueous phases (b) of these three thiols through theoretical calculation. The result was that the attacking ability of Hcy was weakest after losing protons (Table S1). Furthermore, we attempted to detect other possible reducing species existing in biological samples. The test results indicated that $\mathrm{Fe}^{2+}$ and ascorbate showed no fluorescence enhancement, which suggested that KL-DN displayed excellent selectivity for GSH and Cys detection (Figure S14).

2.4. Reaction Mechanism. As far as we know, the probes with azido were found to be very sensitive sensors for sulfide due to the reduction of an azido to amidogen. There were a few literatures reporting that an azido could be reduced by thiols [10]. Because of this, we were interested in the mechanism 


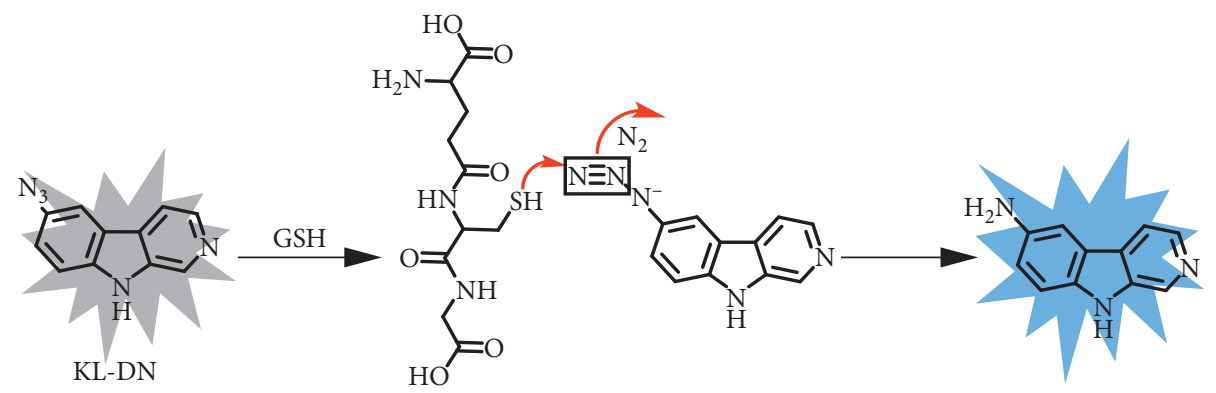

Scheme 1: Chemical structure of KL-DN and the proposed mechanism.

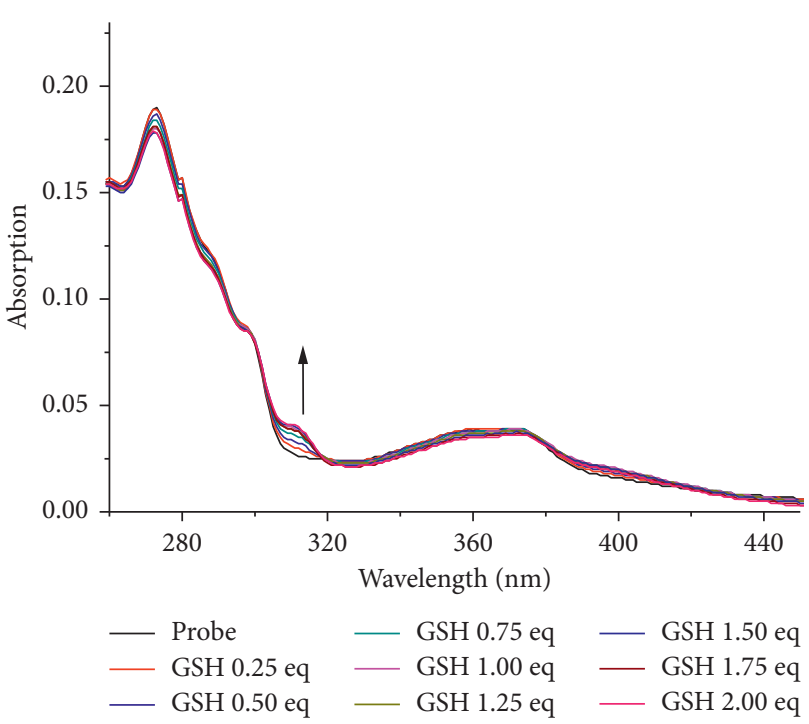

(a)

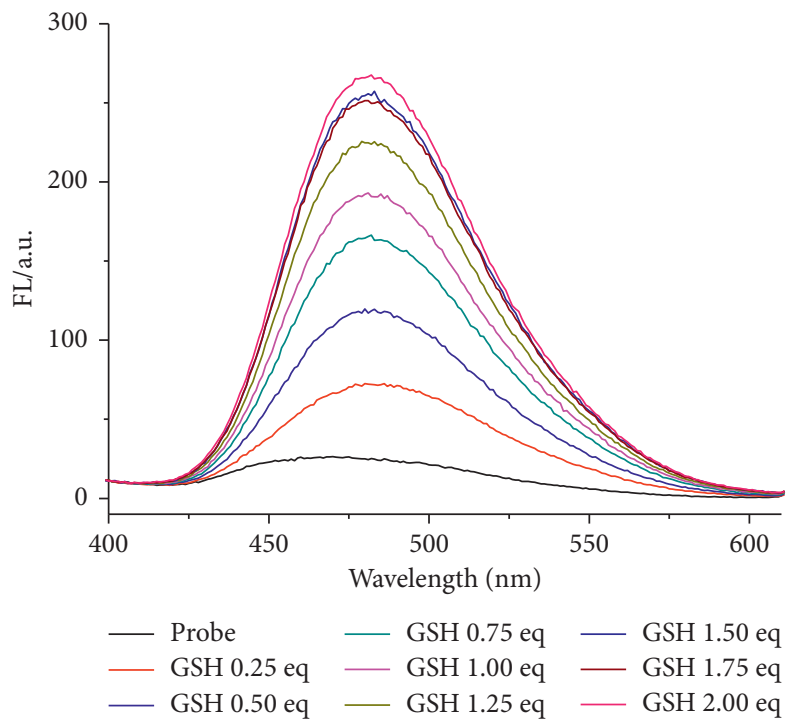

(b)

FIgURE 2: (a) The absorption spectral changes of KL-DN $(10 \mu \mathrm{M})$ upon addition of 2 eq GSH. (b) The fluorescence spectral changes of KLDN $(10 \mu \mathrm{M})$ upon addition of GSH, $\lambda_{\mathrm{ex}}=310 \mathrm{~nm}$. All the solutions are in DMSO/PBS buffer (1:1, v/v, $\left.\mathrm{pH} 7.4\right)$.

and devoted to researching the product from the reaction between KL-DN and GSH. The results of nuclear magnetic titration indicated that no substitution on the aromatic ring was observed upon the gradual addition of GSH. As shown in Figure S15, the obvious upfield shifts from $8.1 \mathrm{ppm}$ to $7.95 \mathrm{ppm}$ for the aromatic protons near the azido were obtained on account of the reduction of azido. And then, we preformed the chemical reaction of KL-DN-GSH and KLDN-Cys with the result of the formation of compound 4 detected by TLC. Next, high-performance liquid chromatography analysis (HPLC) also showed that there was the same peak with the same retention time between KL-DNGSH and compound $\mathbf{4}$ under the same condition (Figure S16). The result of HPLC displayed that there was no new peak after addition of Hcy (Figure S17), which illustrated that Hcy was unable to lead to reduction of KL-DN within a short time. The LC-MS spectrometry was used to confirm the product of KL-DN-GSH and KL-DN-Cys (Figures S18-S21). The molecular weight of the product with retention time of $15.70 \mathrm{~min}$ was the same as the molecular weight of compound 4, which was in accord with the mechanism of the reported literature and the result of HPLC. Subsequently, we confirmed the feasibility of the reaction through the density functional theory (DFT) calculation. The energy gaps of KL-DN between LUMO and HOMO were smaller than compound 4 (Figure S22), revealing that KL-DN was capable of transforming into compound 4.

2.5. Imaging of GSH Presence in HeLa Cells. In order to show the advantage of KL-DN, a research was undertaken to examine the ability of KL-DN to act as an imaging agent for endogenous GSH in HeLa cells. As shown in Figure 4, HeLa cells were incubated with $40 \mu \mathrm{M} \mathrm{KL}-\mathrm{DN}$ for $3 \mathrm{~h}$ at $37^{\circ} \mathrm{C}$ and subsequently washed with PBS ( $\mathrm{pH}$ 7.4). As the negativecontrol experiment, HeLa cells were preincubated with $5 \mathrm{mMN}$-ethylmaleimide (NEM) at $37^{\circ} \mathrm{C}$ for $1 \mathrm{~h}$ to scavenge thiols in the HeLa cells, and then the cells were washed with PBS to remove excess NEM, followed by incubation with $40 \mu \mathrm{M}$ KL-DN for $3 \mathrm{~h}$. In blank-control experiment, no fluorescence was obtained (Figure 4(a)). Exposed to KL-DN, $\mathrm{HeLa}$ cells exhibited strong fluorescence (Figure 4(e), without NEM), while there was no evident fluorescence in the negative control cells that were pretreated with NEM 


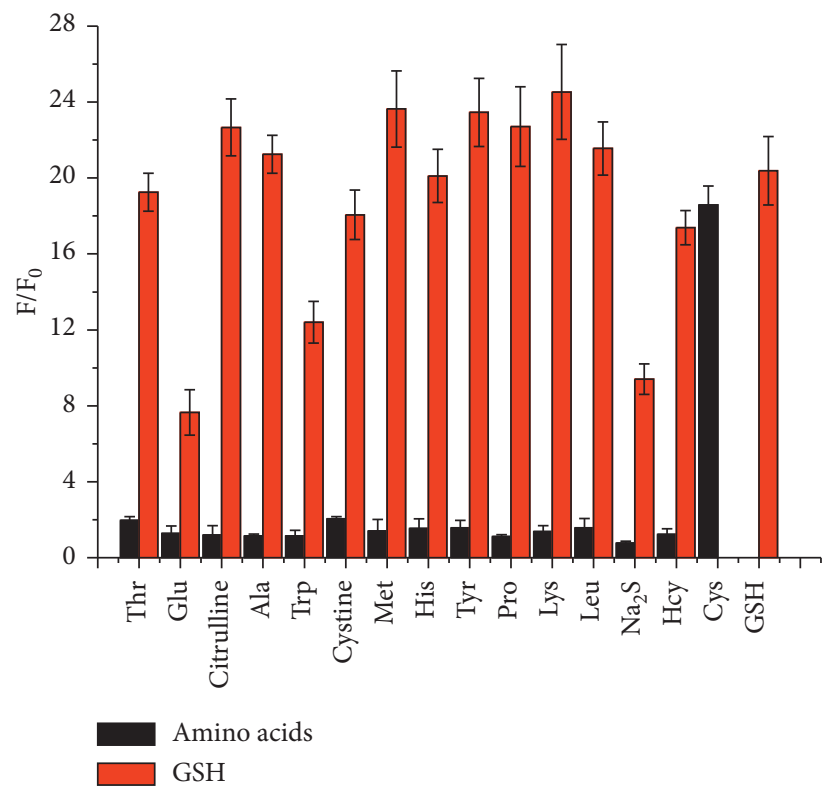

Figure 3: Fluorescence responses of KL-DN $(10 \mu \mathrm{M})$ to different amino acids ( $20 \mu \mathrm{M})$ (blank bar) in DMSO/PBS buffer (1:1, v/v, $10 \mathrm{mM}$, pH 7.4), $\lambda_{\mathrm{ex}}=310 \mathrm{~nm}$. Red bars represent the intensity with subsequent addition of GSH $(20 \mu \mathrm{M})$.
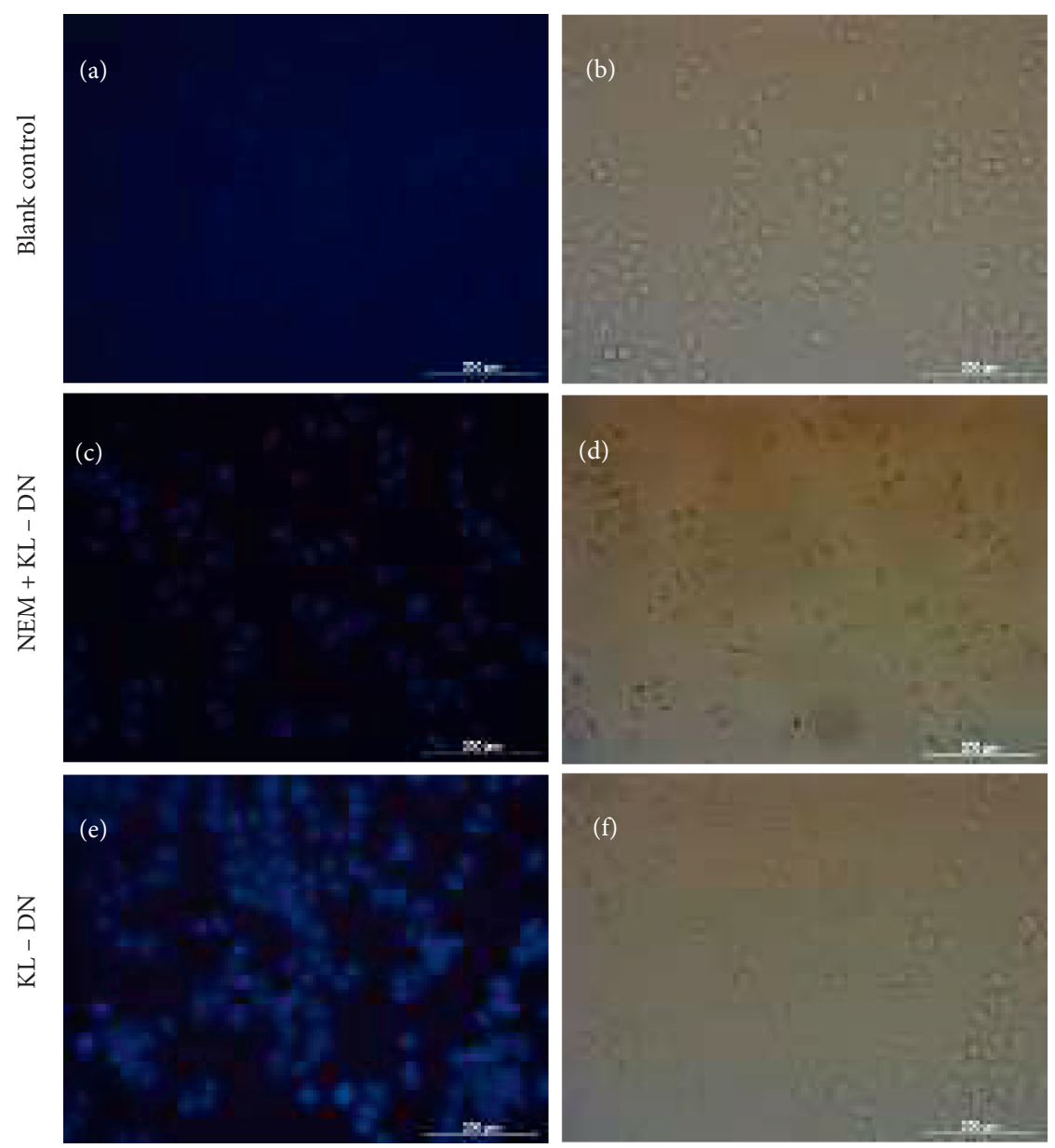

Figure 4: Microscopic images of HeLa cells. (a) Blank control. (b) Corresponding bright field image. (c) Preincubation with 5 mM NEM thiol scavenger for $1 \mathrm{~h}$ at $37^{\circ} \mathrm{C}$, followed by incubation with $40 \mu \mathrm{MKL}-\mathrm{DN}$ for $3 \mathrm{~h}$ at $37^{\circ} \mathrm{C}$. (d) Corresponding bright field image. (e) Incubation for $3 \mathrm{~h}$ at $37^{\circ} \mathrm{C}$ with $40 \mu \mathrm{M} \mathrm{KL}-\mathrm{DN}$. (f) Corresponding bright field image. Scale bar $=200 \mu \mathrm{m}$. 


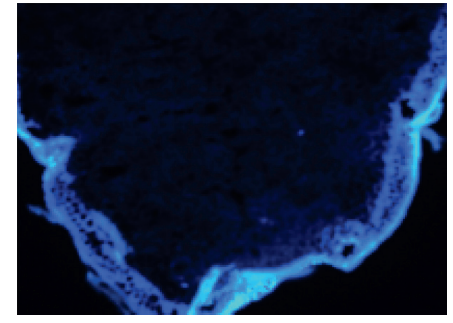

(a)

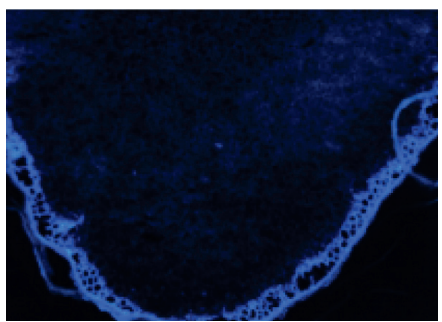

(b)

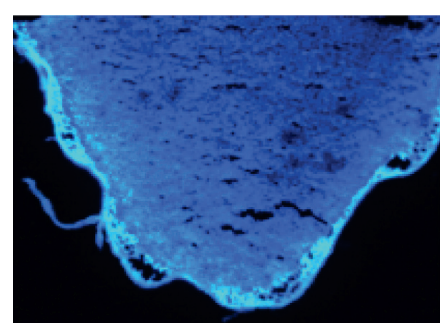

(c)

FIGURE 5: The fluorescent images of wheat germ slice tissues. (a) Blank experiment. (b) The fluorescent images of wheat germ slice tissues pretreated with NEM $(0.5 \mathrm{mM})$ for $5 \mathrm{~min}$ and then incubated with KL-DN $(20 \mu \mathrm{M})$ for $5 \mathrm{~min}$. (c) The fluorescent images of wheat germ slice tissues incubated with KL-DN $(20 \mu \mathrm{M})$ for $5 \mathrm{~min}$. Scale bar $=500 \mu \mathrm{m}$.

(Figure 4(c)). Thus, the electrically neutral KL-DN was able to enter cells and indeed triggered by intracellular GSH in a selective fashion. Furthermore, KL-DN and compound 4 with the skeleton of $\beta$-carboline were necessary to be studied their cytotoxicity against HeLa cells. After modification by azido, KL-DN exhibited low toxicity against HeLa cells after $48 \mathrm{~h}$ via MTT method (Figure S23). Interestingly, compound $\mathbf{4}$ showed negligible toxicity against HeLa cells (Figure S24), which revealed that KL-DN and compound $\mathbf{4}$ had the potential to become imaging agents in cancer cells.

2.6. Fluorescence Imaging of GSH in Wheat Germ Slice Tissues. To further investigate the other biological applications, the fluorescence imaging of KL-DN was carried out in wheat germ slice tissues where abundant GSH exists in wheat germ $(100 \mathrm{mg} / \mathrm{g})$. First of all, the wheat seeds (Xiaoyan 22) were chosen and activated through soaking for $24 \mathrm{~h}$. Then, the sprouted wheat seeds were sliced piece by piece via freezing microtome. The unbroken slices were used for fluorescence imaging. In the control experiment, the wheat germ slice was pretreated with the thiol blocking reagent $\mathrm{N}$-ethylmaleimide (NEM) for $5 \mathrm{~min}$ and then incubated with KL-DN $(20 \mu \mathrm{M})$ for $5 \mathrm{~min}$. The fluorescent microscopy image of the slice showed no fluorescence (Figure 5(b)). The conspicuous fluorescence was observed after adding KL-DN $(20 \mu \mathrm{M})$ (Figure 5(c)). These results indicated that KL-DN was capable of reacting with endogenous GSH with high sensitivity to produce discernible fluorescence responses in the wheat germ tissues.

\section{Conclusion}

In summary, we have designed and synthesized a new 6-azido $\beta$-carboline alkaloid derivative whose fluorescent signal was turned on via redox reaction of quenched group (azido) with biological GSH. With a low limit of detection (92 nM), the probe KL-DN exhibited high selectivity and rapid response toward GSH and Cys when compared to other amino acids and simple gasotransmitter $\mathrm{H}_{2} \mathrm{~S}$. Furthermore, all these features made KL-DN favorable for direct monitoring of GSH in the wheat germ tissues and HeLa cells, demonstrating its practical application in biological systems with high figures of merit. We believe that this new probe will be of great benefit for many researchers engaging in the study of the effect of GSH on plant organisms.

\section{Data Availability}

The data used to support the findings of this study are available from the corresponding author upon request.

\section{Conflicts of Interest}

The authors declare that there are no conflicts of interest regarding the publication of this paper.

\section{Acknowledgments}

This work was supported by the Youth Talent Support Program of Science and Technology Association of Colleges of Shaanxi Province (Grant no. 20190609), Special Scientific Research Plan of Education Department of Shaanxi Province (Grant no. 19JK0190), Open Project of Key Laboratory of Catalytic Foundation and Application of Shaanxi Province (Grant no. SLGPT2019KF01-23), School-Level Project of Shaanxi University of Technology (Grant nos. SLGRC03 and SLGRC02), and Science and Technology Planning Project of Shaanxi Province (Grant no. 2020JM-602).

\section{Supplementary Materials}

Figure S1. Synthetic route. Figure S2. The ${ }^{1} \mathrm{H}$ NMR spectra of compound 4. Figure $S 3$. The ${ }^{13} \mathrm{C}$ NMR spectra of compound 4. Figure S4. The mass spectrum of compound 4. Figure S5. The ${ }^{1} \mathrm{H}$ NMR spectra of KL-DN. Figure S6. The ${ }^{13} \mathrm{C}$ NMR spectra of KL-DN. Figure S7. The mass spectrum of KLDN. Figure S8. (A) The fluorescence intensity change of KL-DN $(10 \mu \mathrm{M})$ at $487 \mathrm{~nm}$ upon addition of GSH, indicative of good linear relationship. (B) The photo of KL-DN in the absence and presence of 2 eq of GSH, which is excited by a handheld UV lamp (365 nm). Figure S9. Job's plot for a $1: 1$ stoichiometry between KL-DN and GSH in DMSO/ PBS buffer ( $1: 1, \mathrm{v} / \mathrm{v}, \mathrm{pH} 7.4), \lambda \mathrm{ex}=310 \mathrm{~nm}$. Figure S10. The effect of $\mathrm{pH}$ from 2 to 12 on the fluorescence signal changes of KL-DN $(10 \mu \mathrm{M})$ at $487 \mathrm{~nm}$ in the absence and presence of 2 equivalents of GSH in DMSO/PBS buffer $(1: 1, \mathrm{v} / \mathrm{v}, \mathrm{pH}$ 7.4), $\lambda$ ex $=310 \mathrm{~nm}$. Figure S11. The absorption spectrum change of KL-DN $(10 \mu \mathrm{M})$ upon addition of 2 equivalents 
of various reducers in DMSO/PBS buffer $(1: 1, \mathrm{v} / \mathrm{v}, 10 \mathrm{mM}$, $\mathrm{pH}$ 7.4). Figure S12. The fluorescence spectral changes of KL-DN $(10 \mu \mathrm{M})$ upon addition of $\mathrm{Na}_{2} \mathrm{~S}(50 \mathrm{eq})$, $\lambda_{\mathrm{ex}}=310 \mathrm{~nm}$. All the solutions are in DMSO/PBS buffer (1: $1, \mathrm{v} / \mathrm{v}, \mathrm{pH}$ 7.4). Figure S13. The fluorescence spectral changes of KL-DN $(10 \mu \mathrm{M})$ upon addition of Hcy (20 eq), $\lambda_{\mathrm{ex}}=310 \mathrm{~nm}$. All the solutions are in DMSO/PBS buffer (1: $1, \mathrm{v} / \mathrm{v}, \mathrm{pH}$ 7.4). Figure S14. The fluorescence intensity change of KL-DN $(10 \mu \mathrm{M})$ at $487 \mathrm{~nm}$ upon addition of 2 equivalents of various reducers in DMSO/PBS buffer $(1: 1$, $\mathrm{v} / \mathrm{v}, 10 \mathrm{mM}, \mathrm{pH} 7.4), \lambda \mathrm{ex}=310 \mathrm{~nm}$. Figure S15. The changes of chemical shift for the aromatic protons of KL-DN upon gradual addition of GSH. Figure S16. HPLC analysis of the KL-DN-GSH, KL-DN-Cys, and compound 4 under the same condition. All the solutions were prefiltered with methanol before HPLC detection. The peaks with retention time of 5.6 and $7.5 \mathrm{~min}$ are assigned to the corresponding fluorescent product, respectively. The retention time differed by 0.2 minutes, which belonged to instrument error. Figure S17. HPLC analysis of the KL-DN, KL-DN-Hcy, and compound 4 under the same condition. Figure S18. LC-MS analysis of the KL-DN-Cys; the solution was preextracted with methanol before LC-MS assay. Figure S19. LC-MS analysis of the KL-DN-Cys with the retention time; the MS spectrum of peak with retention time of $15.76 \mathrm{~min}$, which is assigned to the corresponding fluorescent product. Figure S20. LC-MS analysis of the KL-DN-GSH; the solution was preextracted with methanol before LC-MS assay. Figure S21. LC-MS analysis of the KL-DN-GSH; the MS spectrum of peak with retention time of $15.76 \mathrm{~min}$, which is assigned to the corresponding fluorescent product. Figure S22. Electron orbital energy calculation of KL-DN and compound 4. Figure S23. Cell viability assay of KL-DN incubated in HeLa cell lines for $48 \mathrm{~h}$, and the cell viability was observed via MTT assay. Figure S24. Cell viability assay of compound 4 in HeLa cell lines for $48 \mathrm{~h}$, and the cell viability was observed via MTT assay. Table S1. The NPA charges on $\mathrm{S}$ of $\bullet \mathrm{OH}$ for Path R4 in the gas (a) and aqueous phases (b). (Supplementary Materials)

\section{References}

[1] R. D. Gili, R. M. Torrez Irigoyen, M. C. Penci, S. A. Giner, and P. D. Ribotta, "Wheat germ thermal treatment in fluidised bed. Experimental study and mathematical modelling of the heat and mass transfer," Journal of Food Engineering, vol. 221, pp. 11-19, 2018.

[2] Z.-G. Li, Q. Nie, C.-L. Yang, Y. Wang, and Z.-H. Zhou, "Signaling molecule methylglyoxal ameliorates cadmium injury in wheat (Triticum aestivum L) by a coordinated induction of glutathione pool and glyoxalase system," Ecotoxicology and Environmental Safety, vol. 149, pp. 101-107, 2018.

[3] J. Zhang, X. Ji, J. Zhou, Z. Chen, X. Dong, and W. Zhao, "Pyridinium substituted BODIPY as NIR fluorescent probe for simultaneous sensing of hydrogen sulphide/glutathione and cysteine/homocysteine," Sensors and Actuators B: Chemical, vol. 257, pp. 1076-1082, 2018.

[4] H. Ma, X. Li, X. Liu et al., "Fluorescent glutathione probe based on $\mathrm{MnO} 2-\mathrm{Si}$ quantum dots nanocomposite directly used for intracellular glutathione imaging," Sensors and Actuators B: Chemical, vol. 255, pp. 1687-1693, 2018.

[5] K. Hou, D. Bao, and C. Shan, "Cerium improves the vase life of Lilium longiflorum cut flowers through ascorbate-glutathione cycle and osmoregulation in the petals," Scientia Horticulturae, vol. 227, pp. 142-145, 2018.

[6] F. Cao, E. Ju, C. Liu, F. Pu, J. Ren, and X. Qu, "Coupling a DNA-ligand ensemble with Ag cluster formation for the labelfree and ratiometric detection of intracellular biothiols," Chemical Communications, vol. 52, no. 29, pp. 5167-5170, 2016.

[7] Q. Xiao, H. Gao, Q. Yuan, C. Lu, and J.-M. Lin, "High-performance liquid chromatography assay of cysteine and homocysteine using fluorosurfactant-functionalized gold nanoparticles as postcolumn resonance light scattering reagents," Journal of Chromatography A, vol. 1274, pp. 145-150, 2013.

[8] P. Hess and D. E. Wells, "Evaluation of dialysis as a technique for the removal of lipids prior to the GC determination of ortho- and non-ortho-chlorobiphenyls, using 14C-labelled congeners," The Analyst, vol. 126, no. 6, pp. 829-834, 2001.

[9] Y. Liu, D. Yu, S. Ding, Q. Xiao, J. Guo, and G. Feng, "Rapid and ratiometric fluorescent detection of cysteine with high selectivity and sensitivity by a simple and readily available probe," ACS Applied Materials \& Interfaces, vol. 6, no. 20, pp. 17543-17550, 2014.

[10] J.-Y. Xie, C.-Y. Li, Y.-F. Li et al., "Near-Infrared fluorescent probe with high quantum yield and its application in the selective detection of glutathione in living cells and tissues," Analytical Chemistry, vol. 88, no. 19, pp. 9746-9752, 2016.

[11] Y. Dong, P. Du, M. Pei, and P. Liu, "Design, postpolymerization conjugation and self-assembly of a di-block copolymer-based prodrug for tumor intracellular acid-triggered DOX release," Journal of Materials Chemistry B, vol. 7, no. 37, pp. 5640-5647, 2019.

[12] N. Iglesias, E. Galbis, L. Romero-Azogil et al., "Experimental model design: exploration and optimization of customized polymerization conditions for the preparation of targeted smart materials by the Diels Alder click reaction," Polymer Chemistry, vol. 10, no. 40, pp. 5473-5486, 2019.

[13] Q. Meng, H. Hu, L. Zhou et al., "Logical design and application of prodrug platforms," Polymer Chemistry, vol. 10, no. 3, pp. 306-324, 2019.

[14] D. Zhang, L. Li, X. Ji, and Y. Gao, "Intracellular GSH-responsive camptothecin delivery systems," New Journal of Chemistry, vol. 43, no. 47, pp. 18673-18684, 2019.

[15] X. Wang, J. Lv, X. Yao et al., "Screening and investigation of a cyanine fluorescent probe for simultaneous sensing of glutathione and cysteine under single excitation," Chemical Communications, vol. 50, no. 97, pp. 15439-15442, 2014.

[16] D. Lee, K. Jeong, X. Luo et al., "Near-infrared fluorescent probes for the detection of glutathione and their application in the fluorescence imaging of living cells and tumor-bearing mice," Journal of Materials Chemistry B, vol. 6, no. 17, pp. 2541-2546, 2018.

[17] L. Wang, H. Chen, H. Wang et al., "A fluorescent probe with high selectivity to glutathione over cysteine and homocysteine based on positive effect of carboxyl on nucleophilic substitution in CTAB," Sensors and Actuators B: Chemical, vol. 192, pp. 708-713, 2014.

[18] F. Chen, J. Zhang, W. Qu et al., "Development of a novel benzothiadiazole-based fluorescent turn-on probe for highly selective detection of glutathione over cysteine/homocysteine," Sensors and Actuators B: Chemical, vol. 266, pp. 528-533, 2018. 
[19] S. Lee, J. Li, X. Zhou, J. Yin, and J. Yoon, "Recent progress on the development of glutathione (GSH) selective fluorescent and colorimetric probes," Coordination Chemistry Reviews, vol. 366, pp. 29-68, 2018.

[20] J. Huang, Y. Chen, J. Qi et al., "A dual-selective fluorescent probe for discriminating glutathione and homocysteine simultaneously," Spectrochimica Acta PART A: Molecular and Biomolecular Spectroscopy, vol. 201, pp. 105-111, 2018.

[21] Y. Lin, Y. Tao, F. Pu, J. Ren, and X. Qu, "Combination of graphene oxide and thiol-activated DNA metallization for sensitive fluorescence turn-on detection of cysteine and their use for logic gate operations," Advanced Functional Materials, vol. 21, no. 23, pp. 4565-4572, 2011.

[22] D. Zhang, Z. Yang, H. Li, Z. Pei, S. Sun, and Y. Xu, "A simple excited-state intramolecular proton transfer probe based on a new strategy of thiol-azide reaction for the selective sensing of cysteine and glutathione," Chemical Communications, vol. 52, no. 4, pp. 749-752, 2016.

[23] Q. Zhang, D. Yu, S. Ding, and G. Feng, "A low dose, highly selective and sensitive colorimetric and fluorescent probe for biothiols and its application in bioimaging," Chemical Communications, vol. 50, no. 90, pp. 14002-14005, 2014.

[24] B. Zhu, X. Zhang, H. Jia, Y. Li, S. Chen, and S. Zhang, "The determination of thiols based using a probe that utilizes both an absorption red-shift and fluorescence enhancement," Dyes and Pigments, vol. 86, no. 1, pp. 87-92, 2010.

[25] J. Li, J. Ge, Z. Zhang et al., "A cyanine dye-based fluorescent probe as indicator of copper clock reaction for tracing $\mathrm{Cu}^{2+}$ catalyzed oxidation of cysteine," Sensors and Actuators B: Chemical, vol. 296, pp. 126578-126584, 2019.

[26] W. Chen, X. Yue, H. Zhang et al., "Simultaneous detection of glutathione and hydrogen polysulfides from different emission channels," Analytical Chemistry, vol. 89, no. 23, pp. 12984-12991, 2017.

[27] H. Zhang, L. Xu, W. Chen et al., "A lysosome-targetable fluorescent probe for simultaneously sensing cys/hcy, GSH, and $\mathrm{H}_{2} \mathrm{~S}$ from different signal patterns," ACS Sensors, vol. 3, no. 12, pp. 2513-2517, 2018.

[28] J. Chen, X. Jiang, C. Zhang et al., "Reversible reaction-based fluorescent probe for real-time imaging of glutathione dynamics in mitochondria," ACS Sensors, vol. 2, no. 9, pp. 1257-1261, 2017.

[29] J. Chen, X. Jiang, S. L. Carroll, J. Huang, and J. Wang, "Theoretical and experimental investigation of thermodynamics and kinetics of thiol-michael addition reactions: a case study of reversible fluorescent probes for glutathione imaging in single cells," Organic Letters, vol. 17, no. 24, pp. 5978-5981, 2015.

[30] W. Shen, J. Ge, S. He et al., "A self-quenching system based on bis-naphthalimide: a dual two-photon-channel GSH fluorescent probe," Chemistry-An Asian Journal, vol. 12, no. 13, pp. 1532-1537, 2017.

[31] W. Horton, A. Sood, S. Peerannawar et al., "Synthesis and application of $\beta$-carbolines as novel multi-functional antiAlzheimer's disease agents," Bioorganic \& Medicinal Chemistry Letters, vol. 27, no. 2, pp. 232-236, 2017.

[32] G. M. Olmedo, L. Cerioni, M. M. González, F. M. Cabrerizo, V. A. Rapisarda, and S. I. Volentini, "Antifungal activity of $\beta$-carbolines on Penicillium digitatum and Botrytis cinerea," Food Microbiology, vol. 62, pp. 9-14, 2017.

[33] N. A. Lunagariya, V. M. Gohil, V. Kushwah et al., "Design, synthesis and biological evaluation of 1,3,6-trisubstituted $\beta$-carboline derivatives for cytotoxic and anti-leishmanial potential," Bioorganic \& Medicinal Chemistry Letters, vol. 26, no. 3, pp. 789-794, 2016.

[34] W. Chen, J. Shao, Y. Huang et al., "New $\beta$-carboline fluorophores with superior sensitivity and endoplasmic reticulum specificity for tracking ER changes," Chemical Communications, vol. 55, no. 51, pp. 7327-7341, 2019.

[35] N. Li, J.-K. Dai, H.-T. Du, M.-S. Yuan, J.-W. Zhang, and J.-R. Wang, " $\beta$-Carboline-functionalized dithioacetal as $\mathrm{Hg} 2+$-selective fluorescence probe in water," Spectrochimica Acta Part A: Molecular and Biomolecular Spectroscopy, vol. 136, pp. 900-905, 2015. 